\title{
Analysis of the clinical efficacy of liver resection combined with adjuvant microwave coagulation for patients with hepatocellular carcinoma
}

\author{
Ting Zhang ${ }^{1 \#}$, Mengmeng Wang ${ }^{2 \#}$, Xiangnan Zhang ${ }^{3 \#}$, Jie Hu${ }^{4}$, Weijia Dou ${ }^{1}$, Qingling Fan ${ }^{1}$, Dayun Feng ${ }^{5}$, \\ Lei Liu ${ }^{6}$
}

${ }^{1}$ Department of Nuclear Medicine, The 8th Medical Center of Chinese PLA General Hospital, Beijing 100091, China; ${ }^{2}$ Department of Drug and Equipment, Aeromedicine Identification and Training Centre of Air Force, Xi'an 710069, China; 'Division of Scientific Research, Tangdu Hospital, Fourth Military Medical University, Xi'an 710038, China; ${ }^{4}$ Department of Clinical laboratory, Tangdu Hospital, Fourth Military Medical University, Xi' an 710038, China; ${ }^{5}$ Department of Surgery, Tangdu Hospital, Fourth Military Medical University, Xi'an 710038, China; ${ }^{6}$ Department of Gastroenterology, Tangdu Hospital, Fourth Military Medical University, Xi'an 710038, China

Contributions: (I) Conception and design: L Liu; (II) Administrative support: D Feng; (III) Provision of study materials or patients: M Wang; (IV) Collection and assembly of data: X Zhang; (V) Data analysis and interpretation: J Hu, W Dou, Q Fan; (VI) Manuscript writing: All authors; (VII) Final approval of manuscript: All authors.

\#These authors contributed equally to this work.

Correspondence to: Lei Liu, MD, PhD. Department of Gastroenterology, Tangdu Hospital of the Fourth Military Medical University, No. 569 Xinsi Road, Xi'an 710038, China. Email: tdliulei@fmmu.edu.cn; Dayun Feng, MD, PhD. Department of Surgery, Tangdu Hospital, Fourth Military Medical University, No. 569 Xinsi Road, Xi’an 710038, China. Email: tdfengdy@fmmu.edu.cn.

Background: Liver resection has been widely applied as a curative measure in the treatment of hepatocellular carcinoma (HCC) patients. However, the high rate of postoperative recurrence observed following liver resection proposes a problem, the solution for which is yet to be well established. Microwave coagulation is a therapy that was recently proposed as an adjuvant tool. In this study, we intended to evaluate the effectiveness of microwave coagulation as an auxiliary therapeutic method for patients undergoing liver resection.

Methods: A total of 236 consecutive patients classified as Barcelona Clinic Liver Cancer (BCLC) stage A who had only one tumor were enrolled in this retrospective study, regardless of tumor size. Survival analyses were performed using the Kaplan-Meier method, and the statistical differences between patients who underwent liver resection with and without adjuvant microwave coagulation were examined by the log-rank test. To investigate the prognostic factors for OS, we carried out univariate and multivariate Cox regression analyses.

Results: Based on the Kaplan-Meier curves, patients who underwent surgical resection with intraoperative adjuvant microwave coagulator had prolonged recurrence-free survival time and showed better overall survival (OS) than those who underwent surgical resection alone, with OS at 1, 3, and 5 years of $77.8 \%$, $33.2 \%, 12.6 \%$ vs. 58.2\%, 15.5\%, 9.7\%, respectively (log-rank $\mathrm{P}<0.001$ ). The univariate and multivariate analyses demonstrated that tumor size, albumin, bilirubin, Child-Pugh score, and treatment method had significant prognostic power for both PFS and OS. According to the subgroup analyses based on the tumor size, there were significant differences in PFS and OS among overall subsets between the liver resection with adjuvant microwave coagulator and liver resection only groups.

Conclusions: Liver resection combined with intraoperative adjuvant microwave coagulation had a better prognostic performance than treatment with liver resection alone. Adjuvant microwave coagulation should be suggested as an alternative treatment modality for BCLC stage A patients with a single tumor, regardless of its size.

Keywords: Liver resection; microwave coagulation; hepatocellular carcinoma (HCC) 
Submitted Jan 14, 2020. Accepted for publication Feb 12, 2020.

doi: $10.21037 /$ atm.2020.02.111

View this article at: http://dx.doi.org/10.21037/atm.2020.02.111

\section{Introduction}

As the seventh most frequent type of primary malignant tumor, liver cancer is the third biggest contributor to the global cancer mortality rate (1). Hepatocellular carcinoma (HCC) represents more than $90 \%$ of liver cancer cases (2). The Barcelona Clinic Liver Cancer (BCLC) staging system, due to its notable advantage in prognostic prediction, has been widely adopted as a treatment in clinical practice $(3,4)$. With the development of diagnostic modalities, an increasing number of HCC patients are diagnosed at a very early or early stage of the disease, at which point curative treatments (resection, ablation, and liver transplant) are considered to be the preferential treatments $(5,6)$. However, the high rate of postoperative recurrence observed following liver resection proposes a problem, the solution for which is yet to be well established (7). Previous studies have shown auxiliary therapeutic measures such as postoperative adjuvant transarterial chemoembolization (TACE) to potentially hold benefits for the survival of HCC patients $(8,9)$.

Microwave tissue coagulation has been applied as a therapy which could promote tumor tissue destruction based on a heat-generation mechanism $(10,11)$. Furthermore, the functions of hemostasis and immunization could make it an assistant tool in the process of liver resection. Although adjuvant microwave coagulation could possibly provide an alternative method for patients undergoing liver resection and could observably shorten operation time and reduce intraoperative bleeding, whether it holds survival benefits when used to treat patients who undergo liver resection remains to be fully clarified.

Our study set out to make comparisons between the outcomes of patients who underwent liver resection with and without adjuvant microwave coagulation therapy. Prognostic factors related to recurrence-free survival and overall survival (OS) for patients with single nodular HCC were also identified.

\section{Methods}

\section{Study design and participants}

This retrospective study included consecutive HCC patients who underwent liver resection combined with microwave coagulator or liver resection alone at our department in the period from January 2007 to December 2017. Patients who met the following criteria were included in the study: (I) patients classified as BCLC stage A with a single tumor; (II) no previous therapy for HCC; and (III) no cardiopulmonary, renal, or cerebral dysfunction. Patients who met any of the following criteria were excluded: (I) patients with other uncontrolled ascites, hepatic encephalopathy; and (II) simultaneous malignancies of other system.

HCC was diagnosed by contrast-enhanced magnetic resonance imaging (MRI) and computed tomography (CT) in line with the guidelines of the American Association for the Study of the Liver Disease or European Association for the Study of Liver disease (AASLD/EASL) $(12,13)$. Clinical, laboratory, and imaging data from the enrolled patients were collected from the hospital database. Given the retrospective nature of the study, the requirement to obtain informed consent was waived. This investigation received approval from the the Clinical Research Ethics Committee of the Tangdu Hospital and was performed according to the Declaration of Helsinki.

\section{Treatment and follow-up}

General anesthesia was administered to patients before hepatectomy was performed via a subcostal incision on the right side. The perihepatic ligaments and adhesion tissue were separated to insure abdominal exploration to be carried out. Liver tumors were assessed with intraoperative ultrasound to establish their number, size, distribution, and invasion of adjacent structures, as well as their relationship with vessels, bile ducts, and other structures. The Pringle maneuver technique was used to interrupt hepatic blood flow. The hepatoduodenal ligament was fastened with a rubber tourniquet. The patients underwent either hepatic lobectomy, hepatic segmentectomy, semi-hepatic resection, or partial resection depending on the location, size, and number of the tumors. All hepatectomies were carried out in line with the standard methods advised by the guidelines. Each nodule was removed and passed on to the pathology department.

For the microwave coagulation procedure, the resection range was determined through the use of intraoperative 
ultrasound. A microwave ablation needle was inserted about $4 \mathrm{~cm}$ along with the proposed line of excision marked by electrotome. The interval of the insertion point was $15 \mathrm{~mm}$ while output power was set at $80 \mathrm{~W}$ and 2,450 MHz. Microwave coagulation required about 10-20 seconds. Radiation was not detected at a distance of $3-5 \mathrm{~cm}$ when the microwave coagulator equipment was started.

Each patient was followed up at 1 month following hepatectomy, then at 3-month intervals during the first year, and every 3-6 months in subsequent years accordingly. At every follow-up, patients were routinely examined, which involved physical examinations, blood tests (serum $\alpha$-fetoprotein level, serum biochemistry, and liver biochemistry), and imaging examinations (chest X-ray, abdominal ultrasonography, and abdominal CT or MRI).

\section{Statistical analysis}

Categorical variables are expressed as frequencies. Continuous variables are reported as medians and interquartile ranges. Comparisons of the baseline characteristics of the enrolled patients to identify differences between two groups were carried out using the chi-square test, or for categorical or continuous variables the Fisher's exact test and Mann-Whitney U test were used, respectively. RFS was defined as the period of time between the date of treatment to any recurrence, while OS was defined as the time between the date of liver resection with or without adjuvant microwave coagulation until death or the last follow-up. The last visit took place on March, 2019. Survival analyses were performed using the KaplanMeier method and statistical differences between the liver resection only group and the liver resection with adjuvant microwave coagulation group were examined by the logrank test. The Cox proportional hazards regression model was applied to calculate the hazard ratio (HR) for survival and the $95 \%$ confidence intervals (CI) of prognostic factors for RFS or OS based on the univariate and multivariate analyses. Two multivariate models with stepwise methods for selecting the independent prognostic factors were performed separately to avoid collinearity: model 1, including the baseline characteristics but excluding the Child-Pugh score; and model 2, including the Child-Pugh score and baseline characteristics without albumin and bilirubin. Statistical significance was taken as a two-sided $\mathrm{P}$ value $\leq 0.05$ for all analyses. IBM SPSS software version 23.0 (SPSS Inc., Chicago, IL, USA) facilitated the statistical analyses.

\section{Results}

\section{Baseline characteristics}

A total of 236 consecutive patients classified as BCLC stage A with single nodular HCC who met our inclusion criteria were enrolled in this study. Of these patients, the liver resection only group and the liver resection with adjuvant microwave coagulator group comprised 113 and 123 patients, respectively. No significant differences were observed in characteristics between the two groups. The baseline demographic and clinical characteristics are summarized in Table 1.

\section{Survival analyses of patients between RES and RES plus $M W$ group for recurrence-free survival rate}

For the patients who underwent liver resection combined with adjuvant microwave coagulation, 90 patients experienced relapse during a median follow-up period of 44.5 months. However, in the liver resection monotherapy group, 87 patients relapsed, while the median follow-up period reached 61.7 months. Based on the Kaplan-Meier curves, patients treated with liver resection plus adjuvant microwave coagulation showed better OS compared to those who underwent liver resection alone, with recurrencefree survival rates at 1, 3 and 5 years of $79.3 \%, 36.2 \%$, and $9.2 \%$ vs. $57.7 \%, 13.5 \%$, and $6.7 \%$, respectively (log-rank: $\mathrm{P}<0.001)$ (Figure 1).

\section{Univariate and multivariate analyses of recurrence-free survival rates}

Based on the univariate analysis for recurrence-free survival rate, the following factors were associated with survival: tumor size, treatment method, albumin, bilirubin, and Child-Pugh score $(\mathrm{P}<0.05$; Table 2). The factors above were included in multivariate analysis (Table 3). In multivariate model 1, tumor size (HR, 2.791; 95\% CI, 2.324-3.351; $\mathrm{P}<0.001)$, albumin (HR, 0.557; 95\% CI, 0.490-0.634; $\mathrm{P}<0.001$ ), bilirubin (HR, 1.935; 95\% CI, 1.765-2.122; $\mathrm{P}<0.001$ ), and treatment method (HR, 0.002; 95\% CI, $0.001-0.005 ; \mathrm{P}<0.001)$ were identified as independent predictors of recurrence-free survival rate. In multivariate model 2, the independent prognostic factors included tumor size (HR, 1.833; 95\% CI, 1.662-2.022; $\mathrm{P}<0.001)$, ChildPugh score (HR, 3.348; 95\% CI, 2.301-4.872; $\mathrm{P}<0.001$ ), and treatment method (HR, 0.222; 95\% CI, 0.157-0.314; $\mathrm{P}<0.001)$. 
Table 1 The baseline characteristics of study patients

\begin{tabular}{|c|c|c|c|}
\hline Variable & $M W+$ RES group $(n=123)$ & RES group ( $n=113$ ) & $P$ value \\
\hline Age (years) & 58 [45-66] & $52[43-66]$ & 0.475 \\
\hline Age $(<60 / \geq 60)$ & $57 / 66$ & $44 / 69$ & 0.251 \\
\hline $\mathrm{HBsAg}(\mathrm{P} / \mathrm{N})$ & $111 / 12$ & $98 / 15$ & 0.396 \\
\hline AST level (U/L) & 55 [36-83] & $48[33-66]$ & 0.143 \\
\hline Albumin (g/L) & 42.6 [36.9-45.3] & 39.0 [35.8-44.3] & 0.139 \\
\hline Total bilirubin level ( $\mu \mathrm{mol} / \mathrm{L})$ & $16.6[13.2-21.0]$ & $17.8[10.5-27.7]$ & 0.568 \\
\hline Platelet count $\left(\times 10^{9} / \mathrm{L}\right)$ & 207 [162-293] & 217 [179-244] & 0.387 \\
\hline Tumor size (cm) & $9.1[5.2-11.7]$ & $8.8[6.9-11.2]$ & 0.518 \\
\hline BUN & $5.7[4.7-7.0]$ & $6.1[5.1-7.3]$ & 0.250 \\
\hline $\mathrm{Cr}$ & $68.0[59.4-77.9]$ & 69.0 [61.6-77.0] & 0.151 \\
\hline GGT & 95 [42-167] & $88[45-174]$ & 0.647 \\
\hline WBC & $5.56[4.25-7.00]$ & $4.85[3.44-6.43]$ & 0.088 \\
\hline INR & $1.03[0.97-1.12]$ & $1.04[0.98-1.10]$ & 0.074 \\
\hline
\end{tabular}

MW, microwave; RES, resection; ALT, alanine aminotransferase; AST, aspartate aminotransferase; AFP, alpha fetoprotein; BUN, blood urea nitrogen; GGT, gamma-glutamyl transpeptidase; $\mathrm{Cr}$, creatinine; WBC, white blood cell; INR, international normalized ratio

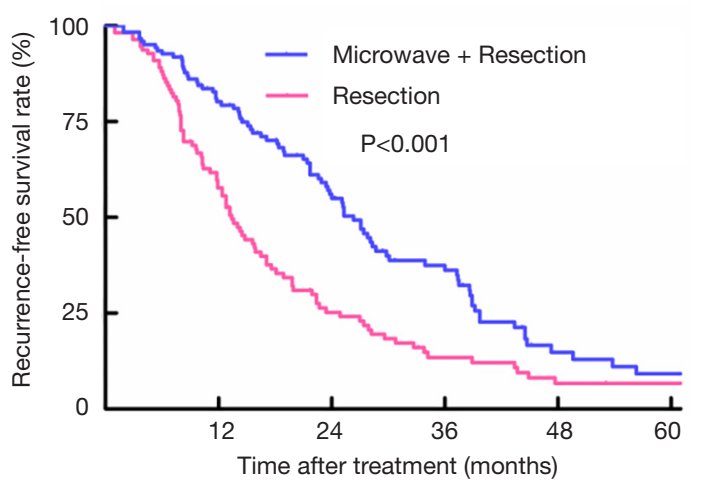

Figure 1 Kaplan-Meier curves for recurrence-free survival (RFS).

\section{Subgroup analysis for recurrence-free survival rate}

Tumor size was further stratified in three different groups to identify whether it influenced the efficacy of HR (Table 4). Regarding patients with tumor size smaller than $5 \mathrm{~cm}$, patients who underwent liver resection in combination with adjuvant microwave coagulation showed a better recurrence-free survival rate compared with those treated through liver resection alone (log-rank: $\mathrm{P}=0.002$ ). For patients with tumor size larger than $5 \mathrm{~cm}$, a significant difference in the recurrence-free survival rate was observed between the two groups (log-rank: $\mathrm{P}<0.001)$.

\section{Survival analyses of patients between RES and RES plus $M W$ group for $O S$}

For patients who underwent liver resection with adjuvant microwave coagulation, 95 patients died during a median follow-up time of 44.2 months. However, in the liver resection group, 86 patients died, and the median followup reached 53.1 months. Based on the Kaplan-Meier curves, patients treated with liver resection with adjuvant microwave coagulator showed a better OS than those undergoing liver resection alone, with $\mathrm{OS}$ at 1,3 , and 5 years of $77.8 \%, 33.2 \%$, and $12.6 \%$ vs. $58.2 \%, 15.5 \%$, and $9.7 \%$, respectively (log-rank: $\mathrm{P}=0.001$ ) (Figure 2 ). 
Table 2 Univariate analysis of prognostic factors of recurrence-free survival rate

\begin{tabular}{|c|c|c|}
\hline \multirow{2}{*}{ Factors } & \multicolumn{2}{|c|}{ Univariate Cox regression } \\
\hline & Hazard ratio (95\% Cl) & $P$ value \\
\hline Sex (male) & $0.824(0.510-1.331)$ & 0.428 \\
\hline Age ( $\geq 60$ years) & $1.178(0.872-1.590)$ & 0.285 \\
\hline Tumor size $(\mathrm{cm})$ & $1.560(1.444-1.685)$ & $<0.001$ \\
\hline AFP (>400 ng/mL) & $0.895(0.659-1.217)$ & 0.481 \\
\hline Albumin (g/L) & $0.756(0.724-0.790)$ & $<0.001$ \\
\hline Total bilirubin level $(\mu \mathrm{mol} / \mathrm{L})$ & $1.394(1.328-1.463)$ & $<0.001$ \\
\hline $\operatorname{PLT}\left(\times 10^{9} / \mathrm{L}\right)$ & $1.001(0.999-1.003)$ & 0.165 \\
\hline AST (units/L) & $0.999(0.997-1.001)$ & 0.463 \\
\hline ALT (units/L) & $0.999(0.995-1.002)$ & 0.526 \\
\hline Child-Pugh score & $2.208(1.572-3.102)$ & $<0.001$ \\
\hline Positive HBsAg & $1.060(0.658-1.708)$ & 0.811 \\
\hline BUN & $1.993(0.974-1.011)$ & 0.440 \\
\hline $\mathrm{Cr}$ & $1.007(0.999-1.016)$ & 0.101 \\
\hline Treatment method & $0.566(0.420-0.763)$ & $<0.001$ \\
\hline GGT & $1.001(0.999-1.002)$ & 0.418 \\
\hline INR & $1.063(0.946-1.194)$ & 0.304 \\
\hline WBC & $0.933(0.864-1.008)$ & 0.079 \\
\hline
\end{tabular}

ALT, alanine aminotransferase; AST, aspartate aminotransferase; AFP, alpha fetoprotein; BUN, blood urea nitrogen; GGT, gamma-glutamyl transpeptidase; $\mathrm{Cr}$, creatinine; WBC, white blood cell; INR, international normalized ratio.

\section{Univariate and multivariate analyses of $O S$}

Based on the univariate analysis for OS, the following factors were associated with survival: tumor size, treatment method, albumin, bilirubin, and Child-Pugh score $(\mathrm{P}<0.05$; Table 5). The factors above were included in multivariate analysis (Table 6). In multivariate model 1 , tumor size (HR, 1.243; 95\% CI, 1.165-1.326; $\mathrm{P}<0.001$ ), bilirubin (HR, 1.079; 95\% CI, 1.050-1.108; $\mathrm{P}<0.001)$, albumin (HR, 0.849; 95\% CI, 0.786-0.873; $\mathrm{P}<0.001)$, and treatment method (HR, 0.639; 95\% CI, 0.467-0.874; $\mathrm{P}<0.001$ ) were identified as being independently predictive of OS. In the multivariate model 2, the independent prognostic factors included tumor size (HR, 1.355; 95\% CI, 1.285-1.428; $\mathrm{P}<0.001$ ), ChildPugh score (HR, 1.753; 95\% CI, 1.295-2.372; $\mathrm{P}<0.001$ ), and treatment method (HR, 0.503; 95\% CI, 0.374-0.676; $\mathrm{P}<0.001)$.

\section{Subgroup analysis for $O S$}

Tumor size was further stratified into three different groups to identify whether it influenced the efficacy of HR (Table 7). Regarding patients with tumor size smaller than $5 \mathrm{~cm}$, those who underwent liver resection plus adjuvant microwave coagulation showed a better OS rate than those treated with liver resection alone (log-rank: $\mathrm{P}=0.004)$. For patients with tumor size exceeding $5 \mathrm{~cm}$, a significant difference was observed in OS rate between the two groups (log-rank: $\mathrm{P}=0.009$ ).

Table 3 Multivariate analysis of prognostic factors of recurrence-free survival rate

\begin{tabular}{|c|c|c|c|c|}
\hline Factors & \multicolumn{2}{|c|}{ Multivariate model 1 for RFS } & \multicolumn{2}{|c|}{ Multivariate model 2 for RFS } \\
\hline Treatment method & $0.002(0.001-0.005)$ & $<0.001$ & $0.222(0.157-0.314)$ & $<0.001$ \\
\hline Albumin (g/L) & $0.557(0.490-0.634)$ & $<0.001$ & - & - \\
\hline Total bilirubin level $(\mu \mathrm{mol} / \mathrm{L})$ & $1.935(1.765-2.122)$ & $<0.001$ & - & - \\
\hline Tumor size $(\mathrm{cm})$ & $2.791(2.324-3.351)$ & $<0.001$ & $1.833(1.662-2.022)$ & $<0.001$ \\
\hline
\end{tabular}


Table 4 Subgroup analyses of prognostic factors of recurrence-free survival rate

\begin{tabular}{lccc}
\hline Variables & $\mathrm{N}(\mathrm{MW}+\mathrm{RES} / \mathrm{RES})$ & Median survival (MW + RES vs. RES) & $\mathrm{P}$ value \\
\hline Tumor size $(\mathrm{cm})$ & & & \\
$<5$ & $59 / 62$ & $38.700 \pm 0.961$ vs. $22.400 \pm 2.064$ & 0.002 \\
$\geq 5$ & $64 / 51$ & $15.100 \pm 0.850$ vs. $11.900 \pm 0.733$ & $<0.001$ \\
\hline
\end{tabular}

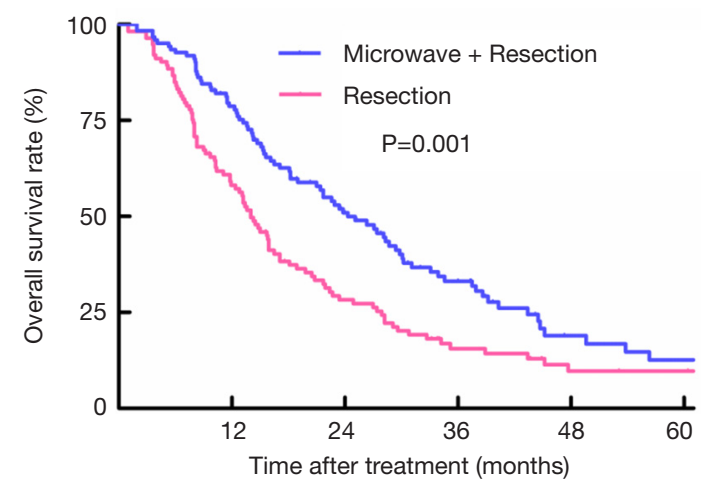

Figure 2 Kaplan-Meier curves for overall survival (OS).

\section{Discussion}

In this retrospective study, we demonstrated in BCLC stage A patients with a single tumor nodule that those who underwent liver resection combined with adjuvant microwave coagulation could prolong recurrence-free survival time and showed a better OS than those who underwent surgical resection alone. Furthermore, treatment method was an independent prognostic indicator of better PFS and OS, while tumor size was an independent predictor of poorer PFS and OS.

On the basis of the Barcelona Clinic Liver Cancer (BCLC) staging system, liver resection is proposed as the standard treatment for HCC patients of BCLC stage A. Nevertheless, given the considerable rate of recurrence, previous studies explored multifarious methods for which the OS is still unsatisfactory.

Previous studies have demonstrated that intraoperative adjuvant microwave coagulation could shorten operation time and reduce intraoperative bleeding, reducing the incidence of complications $(14,15)$. Additionally, the isolation belt caused by microwave coagulation could serve as a barrier to prevent tumors spreading and activate immune cells to play a crucial part in killing tumor cell (16-18). However, as a mass of hepatic cells have died, a relatively higher level of AST was observed in the group treated with intraoperative adjuvant microwave coagulation
Table 5 Univariate analysis of prognostic factors of overall survival

\begin{tabular}{|c|c|c|}
\hline \multirow{2}{*}{ Factors } & \multicolumn{2}{|c|}{ Univariate Cox regression } \\
\hline & Hazard ratio $(95 \% \mathrm{Cl})$ & $P$ value \\
\hline Sex (male) & $0.951(0.608-1.488)$ & 0.826 \\
\hline Age ( $\geq 60$ years) & $1.150(0.855-1.546)$ & 0.355 \\
\hline Tumor size (cm) & $1.343(1.274-1.415)$ & $<0.001$ \\
\hline AFP (>400 ng/mL) & $0.946(0.701-1.277)$ & 0.717 \\
\hline Albumin (g/L) & $0.836(0.812-0.861)$ & $<0.001$ \\
\hline Total bilirubin level $(\mu \mathrm{mol} / \mathrm{L})$ & $1.129(1.109-1.150)$ & $<0.001$ \\
\hline PLT $\left(\times 10^{9} / \mathrm{L}\right)$ & $1.001(0.999-1.003)$ & 0.310 \\
\hline AST (units/L) & $0.999(0.998-1.002)$ & 0.763 \\
\hline ALT (units/L) & $0.999(0.995-1.002)$ & 0.467 \\
\hline Child-Pugh score & $1.853(1.367-2.513)$ & $<0.001$ \\
\hline Positive HBsAg & $1.130(0.694-1.840)$ & 0.624 \\
\hline BUN & $0.991(0.968-1.013)$ & 0.415 \\
\hline $\mathrm{Cr}$ & $0.998(0.990-1.007)$ & 0.732 \\
\hline Treatment method & $0.618(0.461-0.828)$ & 0.001 \\
\hline GGT & $1.001(0.999-1.002)$ & 0.571 \\
\hline INR & $1.071(0.957-1.199)$ & 0.233 \\
\hline WBC & $0.954(0.886-1.027)$ & 0.212 \\
\hline
\end{tabular}

ALT, alanine aminotransferase; AST, aspartate aminotransferase; AFP, alpha fetoprotein; BUN, blood urea nitrogen; GGT, gamma-glutamyl transpeptidase; $\mathrm{Cr}$, creatinine; WBC, white blood cell; INR, international normalized ratio.

which may be eliminated by perioperative careful management. To avoid confounding factors, only patients who had a single tumor who underwent liver resection with or without intraoperative adjuvant microwave coagulation were enrolled in this study. Our present study showed that liver resection combined with intraoperative adjuvant microwave coagulation was more effective than liver resection alone in enhancing prognostic survival in these patients.

Univariate and multivariate analyses were conducted 
Table 6 Multivariate analysis of prognostic factors of overall survival

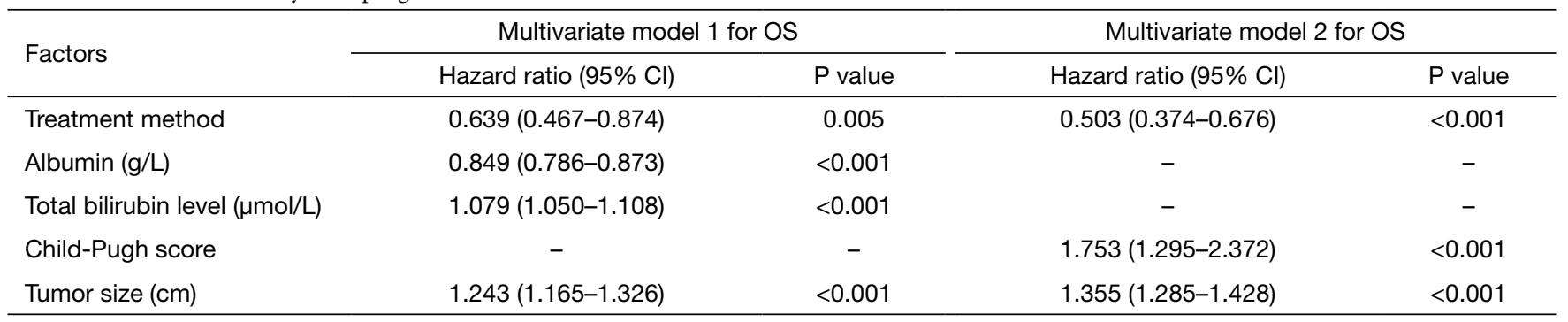

OS, overall survival.

Table 7 Subgroup analyses of prognostic factors of overall survival

\begin{tabular}{lccc}
\hline Variables & N (MW + RES/RES) & Median survival (MW + RES vs. RES) & P value \\
\hline Tumor size $(\mathrm{cm})$ & & & \\
& $59 / 62$ & $37.900 \pm 3.169$ vs. $22.700 \pm 3.782$ & 0.004 \\
$\geq 5$ & $64 / 51$ & $14.200 \pm 0.919$ vs. $11.900 \pm 1.133$ & 0.009 \\
\hline
\end{tabular}

MW, microwave; RES, resection.

to reveal prognostic factors in relation to both RFS and OS. Albumin, bilirubin, Child-Pugh score, tumor size, and treatment method were associated with significant $\mathrm{P}$ values. To address potential collinearity in multivariable analysis, albumin, bilirubin, and Child-Pugh score were included in two different Cox proportional hazards regression models. A relationship was identified between patients with poorer prognosis and larger tumor size and higher bilirubin level. High albumin level was regarded as an indicator of better RFS and OS. Additionally, different treatment methods were shown to be significant independent predictors of RFS and OS. Subgroup analysis revealed that surgical resection plus intraoperative adjuvant microwave coagulation provided a better prognostic performance than surgical resection alone throughout the subsets divided by tumor size. This result demonstrated that intraoperative adjuvant microwave coagulation might be an effective treatment option for BCLC stage A patients with a single tumor, regardless of its size.

However, this study had several limitations that should be discussed. The primary limitation was its retrospective design, which could have introduced information bias. All the procedures and administration were conducted by the same experienced team to ensure quality control and to alleviate potential bias. Additionally, this study was a single center study with a relatively small sample size, which could reduce its representativeness. Further high-quality prospective studies with large sample sizes are needed. Finally, the majority of the subjects in our study were Chinese with hepatitis B virus infection as the cause of HCC, while in most Western countries, the etiologies of HCC are mainly hepatitis $\mathrm{C}$ virus infection and alcoholic liver disease.

In conclusion, this retrospective study demonstrated that liver resection combined with intraoperative adjuvant microwave coagulation provided better prognostic performance than liver resection alone. Adjuvant microwave coagulation should be suggested as an alternative treatment modality for BCLC stage A patients with a single tumor, regardless of its size.

\section{Acknowledgments}

Funding: None.

\section{Footnote}

Conflicts of Interest: All authors have completed the ICMJE uniform disclosure form (available at http://dx.doi. org/10.21037/atm.2020.02.111). LL serves as the unpaid editorial board member of Annals of Translational Medicine from Apr 2020 to Mar 2022. The other authors have no conflicts of interest to declare.

Ethical Statement: The authors are accountable for all aspects of the work in ensuring that questions related to the accuracy or integrity of any part of the work are appropriately investigated and resolved. Given the retrospective nature of the study, the requirement to obtain 
informed consent was waived. This investigation received approval from the Clinical Research Ethics Committee of the Tangdu Hospital and was performed according to the Declaration of Helsinki.

Open Access Statement: This is an Open Access article distributed in accordance with the Creative Commons Attribution-NonCommercial-NoDerivs 4.0 International License (CC BY-NC-ND 4.0), which permits the noncommercial replication and distribution of the article with the strict proviso that no changes or edits are made and the original work is properly cited (including links to both the formal publication through the relevant DOI and the license). See: https://creativecommons.org/licenses/by-nc-nd/4.0/.

\section{References}

1. Bray F, Ferlay J, Soerjomataram I, et al. Global cancer statistics 2018: GLOBOCAN estimates of incidence and mortality worldwide for 36 cancers in 185 countries. CA Cancer J Clin 2018;68:394-424.

2. European Association for the Study of the Liver. EASL Clinical Practice Guidelines: Management of hepatocellular carcinoma. J Hepatol 2018;69:182-236.

3. Tsilimigras DI, Bagante F, Sahara K, et al. Prognosis After Resection of Barcelona Clinic Liver Cancer (BCLC) Stage 0, A, and B Hepatocellular Carcinoma: A Comprehensive Assessment of the Current BCLC Classification. Ann Surg Oncol 2019;26:3693-700.

4. de Freitas LBR, Longo L, Santos D, et al. Hepatocellular carcinoma staging systems: Hong Kong liver cancer vs Barcelona clinic liver cancer in a Western population. World J Hepatol 2019;11:678-88.

5. Li J, Wang J, Lei L, et al. The diagnostic performance of gadoxetic acid disodium-enhanced magnetic resonance imaging and contrast-enhanced multi-detector computed tomography in detecting hepatocellular carcinoma: a meta-analysis of eight prospective studies. Eur Radiol 2019;29:6519-28.

6. Ma H, Sun X, Chen L, et al. Multiplex Immunochips for High-Accuracy Detection of AFP-L3\% Based on SurfaceEnhanced Raman Scattering: Implications for Early Liver Cancer Diagnosis. Anal Chem 2017;89:8877-83.

7. Jung SM, Kim JM, Choi GS, et al. Characteristics of Early Recurrence After Curative Liver Resection for Solitary Hepatocellular Carcinoma. J Gastrointest Surg 2019;23:304-11.

8. Wang L, Ke Q, Deng M, et al. Adjuvant transarterial chemoembolization for patients with hepatocellular carcinoma after radical hepatectomy: a real world study. Scand J Gastroenterol 2019;54:1403-11.

9. Xu D, Liu X, Wang L, et al. Hepatectomy plus adjuvant transcatheter arterial chemoembolization improves the survival rate of patients with multicentric occurrence of hepatocellular carcinoma. Oncol Lett 2018;16:5882-90.

10. Ryu T, Takami Y, Wada Y, et al. Hepatic resection versus operative microwave ablation for single hepatocellular carcinoma $\leq 5 \mathrm{~cm}$ : A propensity score-matched analysis. Surgery 2019;166:254-62.

11. Chong CCN, Lee KF, Chu CM, et al. Microwave ablation provides better survival than liver resection for hepatocellular carcinoma in patients with borderline liver function: application of ALBI score to patient selection. HPB (Oxford) 2018;20:546-54.

12. Bruix J, Sherman M, and American Association for the Study of Liver Diseases. Management of hepatocellular carcinoma: an update. Hepatology 2011;53:1020-2.

13. European Association For The Study Of The Liver and European Organisation For Research And Treatment Of Cancer. EASL-EORTC clinical practice guidelines: management of hepatocellular carcinoma. J Hepatol 2012;56:908-43.

14. Chen YF, Du XL, Lu JG, et al. Application of Microwave Coagulation Therapy in the Irregular Hepatectomy of Patients of Hepatic Carcinoma with Liver Cirrhosis. Progress in Modern Biomedicine 2015;15:2457-61.

15. Chen A, Yang ZY, Lei SX, et al. Application of microwave coagulation in irregular hepatectomy. Modern Oncol 2017;25:1267-71.

16. Imura S, Shimada $M$, Utsunomiya $T$, et al. Ultrasoundguided microwave coagulation assists anatomical hepatic resection. Surg Today 2012;42:35-40.

17. Iida $\mathrm{H}$, Aihara $\mathrm{T}$, Ikuta $\mathrm{S}$, et al. A comparative study of therapeutic effect between laparoscopic microwave coagulation and laparoscopic radiofrequency ablation. Hepatogastroenterology 2013;60:662-5.

18. Eisele RM, Denecke T, Glanemann M, et al. Minimalinvasive microwave coagulation therapy for liver tumours: laparoscopic and percutaneous access. Zentralbl Chir 2014;139:235-43.

Cite this article as: Zhang T, Wang M, Zhang X, Hu J, Dou W, Fan Q, Feng D, Liu L. Analysis of the clinical efficacy of liver resection combined with adjuvant microwave coagulation for patients with hepatocellular carcinoma. Ann Transl Med 2020;8(9):585. doi: 10.21037/atm.2020.02.111 